AN APPARATUS FOR THE INJECTION AND LAVAGE OF THE PELVES OF THE KIDNEYS AND THE URETERS

G. J. THOMAS, M.D.

Physichan to the Muyo Clinle, st. Mary's Hospltal ROCH BSTER, MINN.

In using a syringe or any other apparatus which has a pressure device to foree fluid into the kidneys there is always danger of renal overdistention and colic. In a few instances large hydronephrotic kidneys have been ruptured by this overdistention by the pressure method.

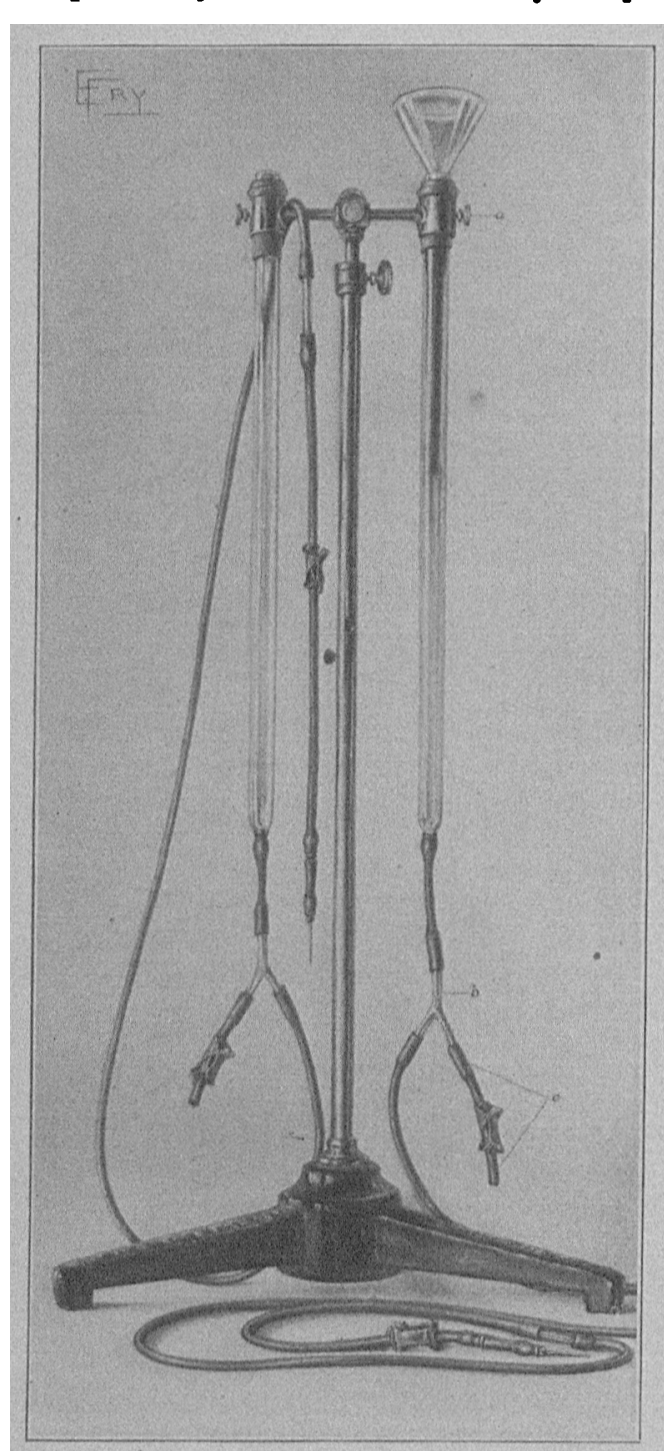

The frequency of o ver d i stention and renal colic $r$ e s u l ting from injecting the pelvis of the kidney and the ureters with shadowcasting fluids by means of a syringe suggested to me the idea of using an apparatus with on l y sufficient force of $\mathrm{gravit} y$ to cause the flow to reach the kidneys.

In $\mathrm{u}$ s i n $g$ the pressure method it is diffieult to control tho a mount of pressure used and the examiner cannot always stop the pressure in time to prevent ove r d is. tention. This crror causes

A ppriatus for the injection and lavage of the pelves of the kildueys and the ureters: $a$, control gerew for ralsing or lowering single buret: $b$, Y-tube; $c$, tube for draining unused colloidal sllver solution.

e patient great annoyance and may cause intense pain for a number of hours following the examination. Moreover, in using the sylinge we are not always able to withdraw the colloidal silver solution from the kidneys and the ureters.

By means of the apparatus here described the pelvis of the kidney can be thoroughly drained of all the fluid which has been injected. 'This procedure is especially helpful in large hydronephroses and pyonephroses, when it is necessury to inject a large quantity of thuid into the kidney.

\section{DESCRIPITON OF APPARATUS}

An ordinary small telescoping irrigating-stand witis a cross-arm at the top of the telescoping rod is used. 'The cross-arm is made to hold two 50 c.c. titrating burets. At the lower end of the buret is a short piece of lubber tubing of small caliber which is connected to a $Y$-shaped glass tube. 'To one arm of the $Y$ from 4 to 5 feet of pressure tubing is attached and to this tubing is attached the needle which is inserted into the lumen of the catheter'. 'T'o the other arm of the $\mathrm{Y}$ is attached 3 or 4 inches of rubber tubing which has a stop-cock at its end. This is used to drain the colloidal silver from the buret before beginning to siphon. In this way the fluid which has not been used can be saved. Water may be poured into the burets to replace the colloidal silver before beginning to siphon. The control screw is used to obtain different heights of the tubes.

We have used this apparatus in the Mayo Clinic in more than fifty injections and in only one case did colic occur. 'This was in a case of stone in the ureter, and the catheter became plugged after passing the stone. 'lhere was a large hydro-ureter with hydronephrosis above the stone, and, since the backed-up urine could not drain off, the colloidal silver suddenly cleared the catheter and caused the temporary pain of overdistention. This was quickly relieved, by lowering the instrument below the level of the patient and siphoning off some of the retained secretion.

In most of the cases in which injection has been employed no measured amount has been used because there is so little pressure that the colloidal silver will outline the pelvis or the ureter and return around the atheter without eausing overdistention.

The pyelograph is taken while the fluid is being injected and the pelvis or the ureter is kept as full as possible at the time the exposure is being made. The instrument works mechanically, and the examiner is not exposed to the $x$-ray while using this method.

The apparatus has been used in cases of double pyelitis when it has been necessary to lavage the pelvis. Both pelves can be washed at one time and the fluid is allowed to run slowly until the patient complains of irritability of the bladder. In this manner irrigation may be continued for a longer period without pain.

The force of gravity only is depended on to cause the flow into the kidneys, the tubes being raised only far enough above the level of the patient to overcome the resistance of tubes and catheters. After allowing a few cubic centimeters of the colloidal silver to run in, the pyelograph is taken while the solution is still rumning, and after this has been completed, the tubes should be lowered at once below the level of the patient, and the colloidal silver siphoned out.

The improvements in technic which we have noted in using this method of injecting the pelves of the kidneys and the ureters are: (1) distention of the pelves of the kidney or ureter or both can be produced without causing colic; (2) a pyelograph can be taken while the pelvis is under the pressure of constantly running fluid, and without exposing the examiner to the $x$-ray; (3) colloidal silver or other solutions can be siphoned off quickly after the plate has been made; and $(t)$ it is useful in slow and prolonged lavage of both renal pelves.

The instrument shown in the drawing is a modified Boehm salvarsan apparatus. An instrument used to inject the kidney should be larger than the Boehm appraratus so that it can rest on the floor and be raised to a height of 6 feet. 